\title{
Radioisotope Distribution Program Progress Report for June 1977
}

\author{
E. Lamb
}




\section{DISCLAIMER}

This report was prepared as an account of work sponsored by an agency of the United States Government. Neither the United States Government nor any agency Thereof, nor any of their employees, makes any warranty, express or implied, or assumes any legal liability or responsibility for the accuracy, completeness, or usefulness of any information, apparatus, product, or process disclosed, or represents that its use would not infringe privately owned rights. Reference herein to any specific commercial product, process, or service by trade name, trademark, manufacturer, or otherwise does not necessarily constitute or imply its endorsement, recommendation, or favoring by the United States Government or any agency thereof. The views and opinions of authors expressed herein do not necessarily state or reflect those of the United States Government or any agency thereof. 


\section{DISCLAIMER}

Portions of this document may be illegible in electronic image products. Images are produced from the best available original document. 
This report was prepared as an account of work sponsored by the United States Government. Neither the United States nor the Energy Research and Development Administration/United States Nuclear Regulatory Commission, nor any of their employees, nor any of their contractors, subcontractors, or their employees, makes any warranty, express or implied, or assumes any legal liability or responsibility for the accuracy, completeness or usefulness of any information, apparatus, product or process disclosed, or represents that its use would not infringe privately owned rights. 
ORNL/TM-6004

Contract No. W-7405-eng-26

OPERATIONS DIVISION

\title{
RADIOISOTOPE DISTRIBUTION PROGRAM
}

PROGRESS REPORT FOR JUNE 1977

\author{
E. Lamb \\ Work Sponsored by \\ ERDA Division of Biomedical and \\ Environmental Research
}

Date Published - August 1977

NOTICE This document contains information of a preliminary nature. It is subject to revision or correction and therefore does not represent a final report.

This report was prepared as an account of work sponsored by the United States Government. Neither the United States nor the United Statest Finerg Research and Development Administration, nor any of

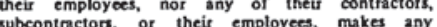
warranty, express or implied, of assumes any legal lisbility or responsibility for the accuracy, completenes or usefulness of any information, apparatus, product of procesv disclosed, or represents that its use would not infringe privately owned rights.

\author{
OAK RIDGE NATIONAL LABORATORY \\ Oak Ridge, Tennessee 37830 \\ operated by \\ UNION CARBIDE CORPORATION \\ for the \\ ENERGY RESEARCH AND DEVELOPMENT ADMINISTRATION
}




\section{THIS PAGE \\ WAS INTENTIONALLY \\ LEFT BLANK}


CONTENTS

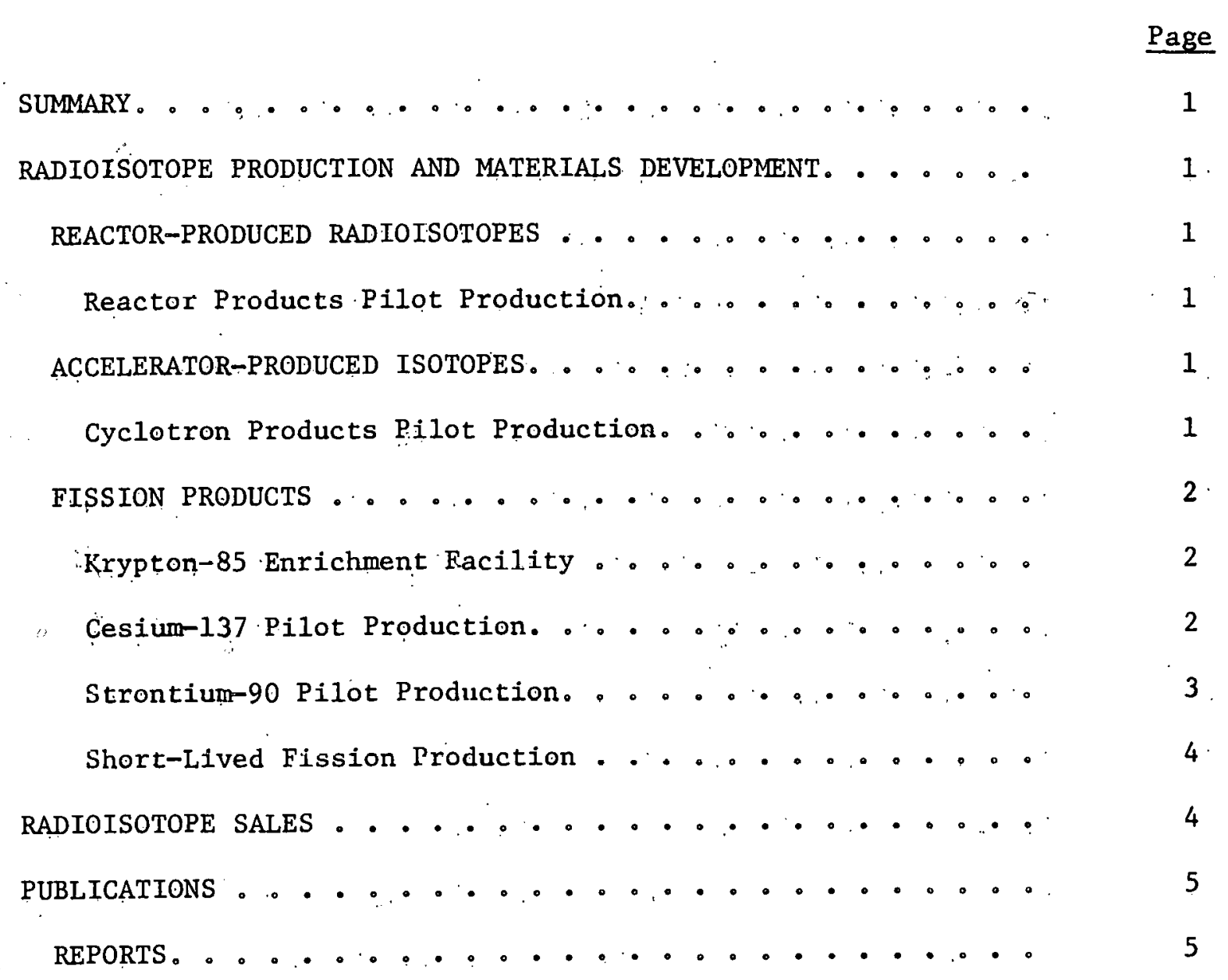




\section{RADIOISOTOPE DISTRIBUTION PROGRAM}

PROGRESS REPORT FOR JUNE 1977

\section{E. Lamb}

\section{SUMIIARY}

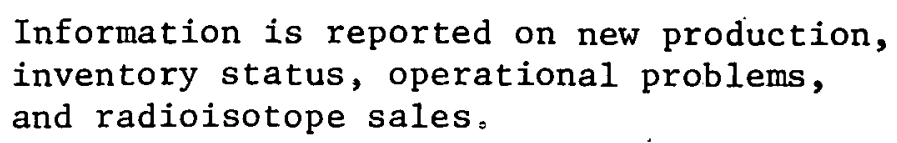

\section{RAD̈IOISOTOPE PRODUCTION AND MATERIALS DEVELOPMENT}

\section{REACTOR-PRODUCED RADIOISOTOPES}

Reactor Products Pilot Production (R. W. Schaich)

(Production and Inventory Accounts)

$\frac{\text { Processed Units }}{\text { Radioisotope }} \frac{\text { Amount (mCi) }}{26}$
Calcium-47
RTOR-PRODUCED ISOTOPES

ACCELERATOR-PRODUCED ISOTOPES

Cyclotron Products Pilot Production (M. R. Skidmore)

(Production and Inventory Accounts)

June 1977 ORNL 86-Inch Cyclotron runs for ORNL and non-ORNL programs are given in Table 1 .

Table 1. Cyclotron Irradiations and Runs for June 1977

\begin{tabular}{cccc}
\hline Date & Product & Target & $\begin{array}{c}\text { Total Time Total } \\
\text { (hr:min) Charges }\end{array}$ \\
\hline
\end{tabular}

\section{ORNL Programs}

\begin{tabular}{|c|c|c|c|c|c|}
\hline $\begin{array}{l}6-18-77 \\
6-26-77\end{array}$ & $\begin{array}{l}\text { ORAU } \\
\text { Y-12 Pl.ant }\end{array}$ & $\begin{array}{l}\text { Carbon-11 } \\
\text { Cobalt }-56\end{array}$ & $\begin{array}{l}\text { Boron. Oxide } \\
\text { Iron }\end{array}$ & $\begin{array}{r}12: 10 \\
6: 15 \\
\end{array}$ & $\begin{array}{r}\$ 1,425 \\
971 \\
\end{array}$ \\
\hline & & & & $18: 25$ & $\$ 2,396$ \\
\hline
\end{tabular}

Non-ORNL Programs

\begin{tabular}{llllll}
$6-15-77$ & New England Nuclear & Cobalt-57 & Nicke1-58 & $51: 15$ & $\$ 9,060$ \\
$6-26-77$ & New England Nuclear & Cobalt-57 & Nicke1-58 & $\frac{51: 15}{102: 30}$ & $\frac{9,090}{\$ 18,150}$ \\
\hline
\end{tabular}




\section{Cyclotron Operations}

Major repairs to the cyclotron were completed June 11, 1977 and regular production runs were started. Malfunction of some components resulted in operation of the cyclotron at a reduced beam current.

FISSION PRODUCTS

Krypton-85 Enrichment Facility (R. W. Schaich)

Fifty percent of the ${ }^{85} \mathrm{Kr}$ enrichment colums were operative during the month of June. A helium leak testing program is still in progress to determine the leak points on the remaining colums, and repairs should be completed by July 1, 1977. Full operation of all the colums should start during July.

Cesium-137 Pilot Production (R. W. Schaich)

(Production and Inventory Accounts)

1. Process Status

Process equipment is in standby status.

2. Operational Summary

\section{Product Inventory}

(Decay calculated through August 31, 1976)

\section{Inventory Material}

Cesium-137 chloride powder

Total Inventory Material
Amount (Ci)

35,450

35,450

Amount (Ci)

Special Form cans

Material returned or stored for customer

$J_{0} \cdot$ L. Shepherd

New England Nuclear Corporation

Puerto Rico sources

Lockheed

AEČL powder

Radiation Resources

Minn. Mining \& Mfg. Company

Gamma Industries

Total Non-Inventory Material

4,500

$$
\begin{array}{r}
3,000 \\
8,100 \\
20,100 \\
54,000 \\
19,700 \\
3,700 \\
8,600 \\
\hline
\end{array}
$$

121,700

TOTAL ' INVENTORY AND NON-INVENTORY MATERIAL 
Fabrication Summary

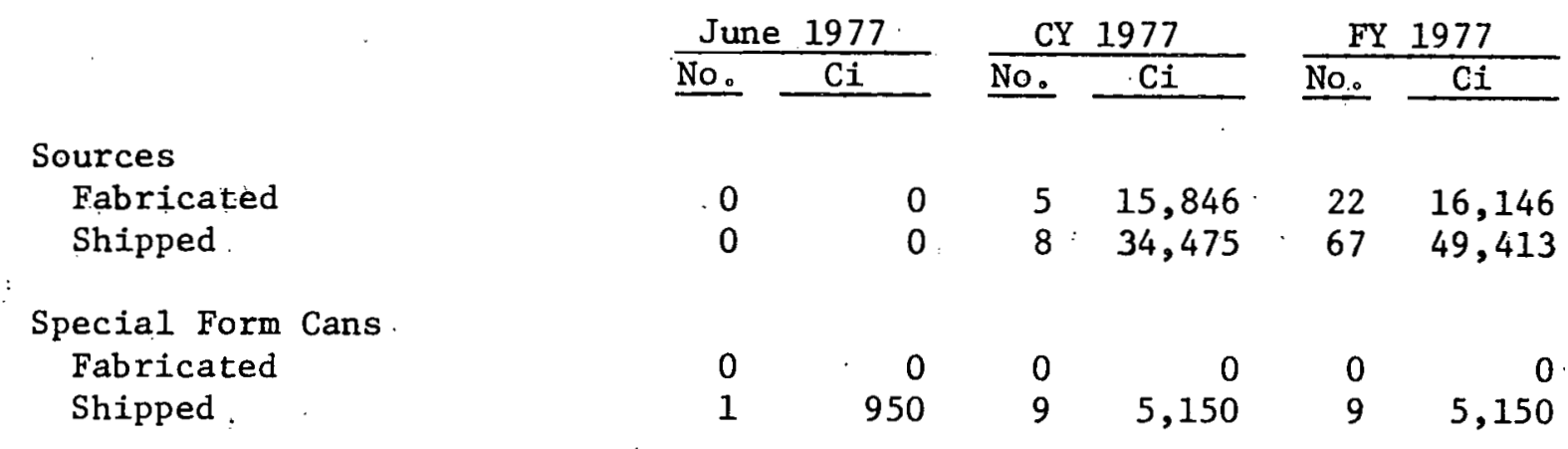

3. Current Orders

All orders on hand have been completed and the material placed into storage awaiting receipt of release for the material.

Strontium-90. Pilot Production (R. W. Schaich)

(Production and Inventory Accounts)

1. Process Status

The ${ }^{90} \mathrm{Sr}$ vacuum hot press testing has been completed and the unit has been installed in the hot cell to fabricate three Sentinel-8 generators for Teledyne. Energy Systems.

\section{Product Inventory}

(Decay calculated through August 31, 1976)

Inventory Material

${ }^{90} \mathrm{Sr}$ titanate powder $( \pm 5 \%)$

Sources in: fabrication

RCA source

${ }^{90} \mathrm{Sr}$ silicate powder (est.)

Stock powder cans

Tatal Inventory Material

Non-Inventory Material

Calorimeter Standards

FPDL recovery material

Quehanna recovery material

Weather Bureau source

SNAP $-7 B$

SNAP $-7 C$.

SNAP - 7D

SNAP material purchase.

Total Non-Inventory Material

TOTAL INVENTORY AND NON-INVENTORY MATERIAL
Amount ( $\mathrm{Ci}$

77,000

57,300

28,000

3,780

166,080

Amount (Ci)

$$
\begin{array}{r}
4,900 \\
19,700 \\
44,000 \\
11,700 \\
160,200 \\
25,200 \\
146,600 \\
254,500 \\
\hline 666,800 \\
\hline 832,880
\end{array}
$$

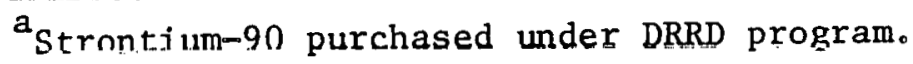




\title{
Fabrication Summary
}

$\frac{\text { June } 1977}{\text { No. } \frac{C i}{\text { No. }} \text { Ci }} \quad \frac{\text { FY } 1977}{\text { No. }}$

\section{Sources}

Fabricated

$\begin{array}{rrrrrr}0 & 0 & 0 & 0 & 0 & 0 \\ 0 & 0 & 1 & 20,000 & 1 & 20,000\end{array}$

Shipped

Special Form.Cans

Fabricated

0

0
10

0

$0 \quad 0$

0

Shipped

1 .

2

20

Short-Lived Fission Production ( $R_{0}, W$. Schaich)

(Production and Inventory Accounts)

\section{Isotope}

Xenon-133
Number of Batches

3
Amount (Ci).

1800

\section{RADIOISOTOPE SALES}

\author{
J. E. Ratzedge
}

Shipments made during the month that may be of interest are listed below:

Customer

Isotope Amount

\section{Large Quantities}

New England Nuclear Corporation

American Atomics Corporation

Brookhaven National Laboratory

Self-Powered Lighting

Saunders-Roe Development, Ltd., England

\begin{abstract}
Tritium
Tritium

Tritium

Tritium

Tritium
\end{abstract}

$$
\begin{array}{r}
10,000 \mathrm{Ci} \\
10,000 \mathrm{Ci} \\
1,500 \mathrm{C1} \\
3,500 \mathrm{Ci} \\
10,000 \mathrm{Ci}
\end{array}
$$

Withdrawn Items
Microbiological Associates
Carbon-14
$0.01 \mathrm{mCi}$

The radioisotope sales and shipments for October 1975 through June 19.76 and the first nine months of fiscal year 1977 are given in Table 2 . 
Table 2. Radioisotope Sales and Shipments

\begin{tabular}{|c|c|c|c|c|}
\hline \multirow{2}{*}{$\begin{array}{l}\quad \text { Item } \\
\text { Inventory items } \\
\text { Major products } \\
\text { Radioisotope services } \\
\text { Cyclotron irradiations } \\
\text { Miscellaneous processed materials } \\
\text { Packing and shipping }\end{array}$} & \multicolumn{2}{|c|}{$\begin{array}{c}10-1-75 \text { thru } \\
6-30-76\end{array}$} & \multicolumn{2}{|c|}{$\begin{array}{c}10-1-76 \text { thru } \\
6-30-77\end{array}$} \\
\hline & $\$$ & $\begin{array}{r}246,437 \\
45,785 \\
111,642 \\
152,977 \\
43,441 \\
95,392 \\
\end{array}$ & $\$$ & $\begin{array}{r}304,279 \\
85,744 \\
150,634 \\
170,551 \\
35,968 \\
145,969 \\
\end{array}$ \\
\hline Total & $\$$ & 695,674 . & $\$$ & 893,145 \\
\hline Number of shipments & & $1, .583$ & & 1,840 \\
\hline
\end{tabular}

\section{PUBLICATIONS}

\section{REPORTS}

E. Lamb, Radioisotope Distribution Program Progress Report for May 1977, ORNL/TM-5979, Oak Ridge National.Laboratory (June 1977)。 
THIS PAGE

WAS INTENTIONALLY

LEFT BLANK 
ORNL / TM-6004

INTERNAL DISTRIBUTION
1. E. E. Beauchamp
2. T。A。Butler
3. F。 No Case.
4. W. R. Casto
5. J. A. Cox
6. $R_{0} \cdot F_{0}$ Hibbs
7. E。 Lamb
8. H: H: Nichol
9. C. L。 Ottinger
10. J. $\mathrm{K}$. Poggenburg
11. H. Postma

\begin{abstract}
12. M。E。 Ramsey
13. J。E. Ratledge

14. C. R。 Richmond

15. R。 Wo Schaich .

16. M. R. Skidmore

17. Mo J. Skinner
\end{abstract}

18-19. Central Research Library

20-21. Laboratory Records Department

22. Laboratory Records - RC

23. Document Reference. Section

\section{EXTERNAL DISTRIBUTION}

24. B..J。 Dropesky, LASL, Los Alamos, New Mexico

25-26. J. Ho Jarrett, PNL, Richland, Washington

27: D. Ko Jones, Richland Operations Office, Richland, Washington

28. J. No. Maddox, ERDA-DBER, Washington, D.C.

29. H. A, O ${ }^{8}$ Brien, LASL, Los Alamos, New Mexico

30. F。 Jo Skozen (Krizek), Argonne Cancer Research Hospital, Chicago

31. Lo Go Stang, Jr., BNL, New. York

32. W: Ho Weyzen, ERDA-DBER, Washington, D。C.

33-34. R。 Wo. Wood, ERDA-DBER; Washington, D.C.

35. Donner Laboratory Library, Univ。 of California, Berkeley, Calif., 94720

36. Research and Technical Support Division, ORO

37-38. Technical Information Center 